\title{
Resultados e indicações de ceratoplastias penetrantes realizadas por médicos em treinamento, num país em desenvolvimento
}

\author{
Indications and results of penetrating Keratoplasty performed by training physicians \\ in a developing country
}

$\begin{array}{llll}\text { Marta } & \text { Ferrari } & \text { Teixeira } & \\ \text { Gildásio } & \text { Castello } & \text { de Almeida } & \mathrm{Jr}^{2} \\ \text { Maria } & \text { Letícia } & \text { Rodrigues } & \\ \text { Paula } & \text { Sayuri } & \text { Kamimoto }^{4} & \\ \text { Luiz } & \text { Kazuo } & \text { Kashiwabuchij } & \end{array}$

${ }^{1}$ Prof. Assistente de Oftalmologia da Faculdade de Medicina de São José do Rio Preto (FAMERP). Mestre em Medicina Interna.

${ }^{2}$ Médico Contratado pelo Hospital de Base - FUNFARME Pós-graduando em Oftalmologia pela Universidade de São Paulo - Ribeirão Preto.

${ }^{3}$ Graduanda do $6^{\circ}$ ano da Faculdade de Medicina de São José do Rio Preto.

${ }^{4}$ Estagiária na Oftalmologia da Faculdade de Medicina de São José do Rio Preto.

${ }^{5}$ Prof. Adjunto Oftalmologia da Faculdade de Medicina de São José do Rio Preto. Doutor em Oftalmologia pela Universidade Federal de Minas Gerais.

Endereço para correspondência: Av. Brigadeiro Faria Lima, 5416 - S. José do Rio Preto (SP) CEP 15090-000. E-mail:martaft@westnet.com.br

\begin{tabular}{|c|}
\hline RESUMO \\
\hline $\begin{array}{l}\text { Objetivos: Avaliar as indicações mais freqüentes, bem como o resul- } \\
\text { tado da acuidade visual final após a ceratoplastia penetrante, realiza- } \\
\text { das por residentes em treinamento. Métodos: Avaliaram-se retrospecti- } \\
\text { vamente } 159 \text { casos de ceratoplastia penetrante realizadas no período } \\
\text { de janeiro de } 1991 \text { a outubro de } 1998 \text {, na Faculdade de Medicina de São } \\
\text { José do Rio Preto - Hospital de Base, correlacionando-os em termos } \\
\text { de indicação para ceratoplastia penetrante e acuidade visual final. O } \\
\text { tempo mínimo de acompanhamento para obtenção da acuidade visual } \\
\text { final foi de seis meses. Para comparação e análise estatística da } \\
\text { acuidade visual final, os casos de ceratoplastia penetrante foram } \\
\text { divididos em seis grupos de diagnóstico pré-operatório: ceratocone, } \\
\text { ceratopatia bolhosa pseudofácica, ceratopatia bolhosa afácica, } \\
\text { distrofia endotelial de Fuchs, ceratopatia em faixa e leucomas. Todas } \\
\text { as cirurgias foram realizadas por residentes em treinamento, sob } \\
\text { supervisão docente. Resultados: O ceratocone foi o grupo que atingiu } \\
\text { um maior aumento médio de acuidade visual pós-operatória em linhas } \\
\text { (7,55 } 2,83) \text { e ceratopatia em faixa o menor }(0,33 \pm 3,20) \text {. Constatou-se } \\
\text { significativa diferença entre as indicações encontradas nos países } \\
\text { desenvolvidos, onde se observa predominância de ceratocone. De } \\
\text { acordo com nosso estudo, as principais indicações para ceratoplastia } \\
\text { penetrante foram, em ordem decrescente, leucoma, ceratopatia bolhosa } \\
\text { pseudofácica, ceratocone, distrofia endotelial de Fuchs, ceratopatia } \\
\text { bolhosa afácica e ceratopatia em faixa. Conclusão: O ceratocone apre- } \\
\text { sentou diferença significativa (p < 0,05) em termos de melhora de } \\
\text { acuidade visual final quando comparado com os outros } 5 \text { grupos de } \\
\text { indicação para ceratoplastia penetrante. }\end{array}$ \\
\hline
\end{tabular}

Descritores: Opacidade da córnea; Ceratoplastia penetrante; Acuidade visual; Países em desenvolvimento; Corpo clínico hospitalar

\section{INTRODUÇ̃̃O}

As indicações de ceratoplastia penetrante $(\mathrm{CP})$ têm sofrido modificações nas últimas décadas e, além disso, apresentam diferenças conforme o país e seu nível sócio-econômico ${ }^{(1-4)}$. As mudanças na freqüência das patologias corneanas, o aprimoramento de técnicas cirúrgicas e instrumental, bem como o implante de lentes intra-oculares tem contribuído para o aumento no número de ceratoplastias penetrantes realizadas nos últimos 
anos e para a maior taxa de sucesso das mesmas ${ }^{(5)}$. Existe pouca informação a respeito da taxa de sucesso de CP realizadas por cirurgiões em treinamento ${ }^{(6)}$. Segundo Abbot, Foster (1979), o diagnóstico pré-operatório e a experiência do cirurgião parecem ser os fatores mais importantes para manter a transparência corneana após a CP comparado com a idade do doador e tempo de enucleação do doador após a morte ${ }^{(7)}$. O transplante de córnea realizado no mundo em desenvolvimento tem apresentado pobres resultados da acuidade visual (AV), principalmente em categorias de pior prognóstico e na presença de algum fator de risco $^{(8)}$.

O presente estudo tem como objetivo avaliar as indicações mais freqüentes, bem como o resultado da acuidade visual final após a ceratoplastia penetrante, realizadas por residentes em treinamento.

\section{MÉTODOS}

Foram analisados retrospectivamente 179 olhos submetidos à CP, realizadas no período de janeiro de 1991 a outubro de 1998 no serviço de oftalmologia da Faculdade de Medicina de São José do Rio Preto - Hospital de Base. Para tal análise, utilizou-se um protocolo baseado no prontuário médico, considerando os seguintes dados: acuidade visual (AV) com correção óptica máxima, biomicroscopia, ceratometria, oftalmoscopia indireta e, quando necessário, para auxiliar o diagnóstico pré-operatório, exames complementares tais como: ecografia, topografia corneana e microscopia especular. Persistindo a dúvida em relação ao diagnóstico clínico, a córnea foi submetida a estudo anatomopatológico. Consideraram-se ainda as complicações per e pós-operatórias, bem como o tempo de seguimento ambulatorial dos pacientes. Todas as córneas doadoras foram preservadas em solução conservante (Optisol-GS) e mantidas em refrigeração, com temperatura média de $4^{\circ} \mathrm{C}$.

Todas as cirurgias foram realizadas sob anestesia geral, após prévia avaliação clínica e cardiológica de cada paciente. O botão doador foi obtido através da trepanação da superfície endotelial contra o bloco de teflon, com diâmetro variando de 7,0 a $8,0 \mathrm{~mm}$. A córnea receptora foi trepanada com mesmo diâmetro ou $0,5 \mathrm{~mm}$ inferior. $\mathrm{O}$ botão doador foi suturado no leito receptor utilizando-se fio mononylon 10.0, com 16 pontos interrompidos ou sutura contínua. Em pacientes com catarata associada, optou-se por cirurgia combinada (ceratoplastia penetrante, facectomia extra-capsular e implante de lente intra-ocular (LIO). As cirurgias foram realizadas por residentes do terceiro ano, supervisionados por um professor responsável).

Das 179 cirurgias realizadas no período, 20 foram excluídas do estudo, pois não obedeciam aos seguintes critérios de inclusão: 1) tempo de seguimento por um período mínimo de seis meses; 2) presença de dados completos no prontuário médico para preenchimento do protocolo e; 3) ceratoplastias penetrantes com finalidade óptica. Os 159 olhos restantes, submetidos a ceratoplastia penetrante, foram divididos em 6 grupos: 1) ceratocone, com idade média de 27,78 $\pm 11,88$ anos; 2) ceratopatia em faixa, com idade média de 73,2 $\pm 5,43$ anos; 3 ) distrofia endotelial de Fuchs, com idade média de 67,46 $\pm 11,75$ anos; 4) ceratopatia bolhosa afácica, com idade média de $65,7 \pm$ 14,67 anos; 5) ceratopatia bolhosa pseudofácica, com idade média de $64,68 \pm 17,18$ e 6) leucomas com idade média de $49,13 \pm 22$ anos. O grupo leucomas foi subdividido em subgrupos, uma vez que, considerou-se leucomas opacidades corneanas resultantes de: a) trauma com perfuração; b) tracoma cicatricial; c) queimadura química; d) ceratite e e) outras.

Para medida da acuidade, foi aplicada a tabela de Snellen ampliada, utilizada por Price et al. ${ }^{(9)}$, considerada a mais apropriada, cujos parâmetros são: $20 / 20=1 ; 20 / 25=2 ; 20 / 30=3$; $20 / 40=4 ; 20 / 50=5 ; 20 / 60=6 ; 20 / 70=7 ; 20 / 80=8 ; 20 / 100=9$; $20 / 200=10 ; 20 / 300=11 ; 20 / 400=12 ;$ contar dedos $=13 ;$ movimento de mãos $=14$; percepção luminosa $=15$ e sem percepção luminosa $=17$, onde cada número corresponde a uma linha de visão. A variação da AV com correção óptica após a $\mathrm{CP}$ pôde então ser quantificada através de mudanças nas linhas de visão, possibilitando assim a comparação entre os diferentes grupos.

Para o estudo estatístico da variação da AV, foi utilizada a análise de variância (ANOVA) seguida do teste de Duncan para comparações múltiplas, adotando um nível de significância igual a 0,05 .

\section{RESULTADOS}

A indicação mais freqüente de CP foi o leucoma $(53,4 \%)$, e a menos foi a ceratopatia em faixa $(5,6 \%)$. A AV pré-operatória, medida pela tabela de Snellen ampliada, foi comparada à $\mathrm{AV}$ pós-operatória e, a evolução desta medida em linhas foi analisada para cada grupo, conforme mostra a tabela 1 .

$\mathrm{O}$ ceratocone foi o grupo que atingiu um maior aumento médio da AV pós-operatória em linhas $(7,55 \pm 2,83)$ e a ceratopatia em faixa o menor $(0,33 \pm 3,20)$. Para os leucomas, a média foi 2,69 4,22. O ceratocone mostrou diferença significativa, em comparação pareada com os outros cinco grupos. Os demais grupos não apresentaram diferença significativa em comparações pareadas, concluindo que as médias em linhas não diferem entre si quando aplicado o teste de Duncan.

Os cinco subgrupos de leucoma foram também avaliados e os resultados são mostrados na tabela 2 .

Entre os cinco subgrupos de leucoma (pós-perfuração, tracoma cicatricial, por queimadura química, pós ceratite e de causa desconhecida) também se observou uma diferença significativa $(\mathrm{p}<0,01)$ entre as médias de mudança da AV em linhas. $\mathrm{O}$ tracoma cicatricial apresentou uma melhora da AV média em linhas igual a cinco, enquanto o subgrupo de queimadura química apresentou piora da AV pós-operatória.

As complicações per e pós-operatórias são apresentadas na tabela 3. A falência de enxerto, extravasamento de sutura (Seidel) e o glaucoma pós-operatório foram as principais complicações. 


\begin{tabular}{|c|c|c|c|c|}
\hline & N. ${ }^{\circ}$ olhos $(\%)$ & $\begin{array}{c}\text { Variação média } \\
\text { da AV (linhas) }\end{array}$ & $\begin{array}{c}\text { Desvio padrão } \\
\text { da AV }\end{array}$ & Tempo (meses) \\
\hline Ceratocone & $18(11,3 \%)$ & $+7,55$ & $\pm 2,83$ & $15,28 \pm 7,3$ \\
\hline Ceratopatia em faixa & $09(5,6 \%)$ & $+0,33$ & $\pm 3,20$ & $11,4 \pm 9,4$ \\
\hline Distrofia endotelial de Fuchs & $15(9,4 \%)$ & $+2,40$ & $\pm 3,02$ & $9,2 \pm 5,93$ \\
\hline Ceratopatia bolhosa pseudofácica & $22(13,8 \%)$ & $+3,36$ & $\pm 3,54$ & $8,18 \pm 2,74$ \\
\hline Ceratopatia bolhosa afácica & $10(6,3 \%)$ & $+2,04$ & $\pm 3,03$ & $9,3 \pm 4,08$ \\
\hline Leucomas & $85(53,4 \%)$ & $+2,69$ & $\pm 4,22$ & $11,57 \pm 6,63$ \\
\hline ANOVA $(p=0,01)$ & & & & \\
\hline
\end{tabular}

\begin{tabular}{|c|c|c|c|}
\hline \multicolumn{4}{|c|}{$\begin{array}{c}\text { Tabela } 2 \text { - Subgrupos de Leucoma: Variação da AV } \\
\text { pós-operatória comparada }\end{array}$} \\
\hline Pré-operatória & $\mathbf{N}^{\circ}$ de olhos & $\begin{array}{l}\text { Variação média } \\
\text { (linhas) }\end{array}$ & $\begin{array}{l}\text { Desvio } \\
\text { padrão }\end{array}$ \\
\hline Pós-perfuração & $19(22,3 \%)$ & $+1,80$ & $\pm 4,26$ \\
\hline Tracoma cicatricial & $07(8,2 \%)$ & $+5,00$ & $\pm 2,31$ \\
\hline Queimadura química & $06(7,0 \%)$ & $-0,16$ & $\pm 2,23$ \\
\hline Pós ceratite & $29(34,1 \%)$ & $+4,45$ & $\pm 4,95$ \\
\hline Causa desconhecida & $24(28,2 \%)$ & $+1,29$ & $\pm 2,90$ \\
\hline \multicolumn{4}{|c|}{ ANOVA $(p<0,01) \quad$ AV $=$ Acuidade Visual } \\
\hline
\end{tabular}

Dos 159 olhos operados, 53 foram submetidos à cirurgia combinada à facectomia com ou sem implante de LIO, sendo que a diferença na média de variação visual em linhas não se mostrou estatisticamente significante nestes casos.

\section{DISCUSSÃO}

A melhora da AV após CP é afetada por um complexo de inúmeros fatores, incluindo a cicatrização corneana e a diminuição gradual do astigmatismo irregular ${ }^{(9)}$. Isto ocorre em taxas variáveis em diferentes indivíduos, mas parece ser influenciada pela idade do receptor, ou seja, quanto mais jovem, mais rapidamente ocorrerá a cicatrização $0^{(9)}$. Portanto a melhora significativa da $\mathrm{AV}$ encontrada no grupo do ceratocone pode ser explicada pelo fator idade, uma vez que este foi o grupo mais jovem. Além disso, pacientes mais jovens teriam menos fatores de morbidade ocular, tais como glaucoma, degeneração macular relacionada à idade e, em cirurgia combinadas, edema macular cistóide recorrente ${ }^{(9)}$. Ao analisarmos os fatores de risco pré-operatórios para a falência do enxerto, tais como a disfunção endotelial, glaucoma, córnea neovascularizada, sinéquia anterior de íris, afacia ou pseudofacia ${ }^{(10)}$, estes praticamente inexistiram no grupo de pacientes com ceratocone e também foram pouco encontrados nos pacientes com tracoma cicatricial. Quando comparamos a melhora da AV com dados da literatura ${ }^{(6-7,9)}$, obtivemos um resultado inferior na mensuração da AV. Contudo para a mensuração da AV final em nosso estudo, não foi utilizada correção com lentes de contato. O resultado da acuidade visual final da queimadura química foi inferior aos outros resultados, já que em países em desenvolvimento, alguns diagnósticos pré-operatórios e certos fatores de risco ocasionam taxa de sobrevida corneana e resultado visual final muito pobre após o transplante de córnea $^{(8)}$. Ainda assim devemos mencionar a escassez de córneas doadoras e a qualidade dos bancos de olhos nos países em desenvolvimento ${ }^{(8)}$, e se com isso, não deveríamos reservar córnea para casos com melhor prognóstico. Nos pacientes com queimadura química da córnea não foi realizado nenhum procedimento antes da $\mathrm{CP}$, como transplante de limbo e transplante de membrana amniótica, o que poderia ocasionar melhores resultados. O tempo de seguimento dos diferentes

\begin{tabular}{|c|c|c|}
\hline Complicações & $\begin{array}{l}\text { Freqüência de complicações } \\
\qquad(n=159)\end{array}$ & $\begin{array}{l}\text { Maior freqüência de cada complicação em } \\
\text { relação ao número amostral de cada grupo }\end{array}$ \\
\hline Falência do enxerto & $18,87 \%$ & $\operatorname{CBP}(27 \%)$ e $\operatorname{DEF}(26,6 \%)$ \\
\hline Extravasamento de sutura (Seidel) & $10,06 \%$ & CF(33\%) e DEF (15\%) \\
\hline Catarata pós-operatória & $1,88 \%$ & $\mathrm{CF}(11 \%)$ \\
\hline Ceratite & $2,5 \%$ & $\operatorname{DEF}(13,3 \%)$ \\
\hline Endoftalmite & $3,14 \%$ & $\mathrm{CF}(11 \%)$ e $\mathrm{CBA}(10 \%)$ \\
\hline Descolamento de retina & $0,63 \%$ & $\mathrm{CBA}(10 \%)$ \\
\hline Glaucoma pós-operatório & $7,54 \%$ & $\mathrm{CBA}(30 \%)$ e $\mathrm{CF}(22 \%)$ \\
\hline Hemorragia expulsiva da coróide & $0,63 \%$ & $\operatorname{LEUC}(1,2 \%)$ \\
\hline Glaucoma maligno & $0,63 \%$ & $\mathrm{CBP}(4,5 \%)$ \\
\hline
\end{tabular}


grupos de diagnóstico pré-operatório não foi o mesmo, Price et al. ${ }^{(9)}$ mostraram que em casos de ceratopatia bolhosa pseudofácica, a AV pós-operatória pode demorar um período maior que um ano para a melhora. Portanto este fato pode ter interferido na $\mathrm{AV}$ final do estudo, como também influenciado negativamente a comparação da AV entre os grupos de diagnósticos pré-operatórios. Os pacientes submetidos à $\mathrm{CP}$, após 5 a 10 anos apresentam uma taxa de perda de células endoteliais maior que a taxa normal, indicando continua instabilidade e disfunção endotelial ${ }^{(11-12)}$. Contudo apesar de ocorrer uma redução da densidade de células endoteliais, a córnea transplantada pode ser mantida viável por até 33 anos após a $\mathrm{CP}^{(13)}$. Assim o tempo final da mensuração da AV para estudo é muito variável, qual seria o tempo ideal para mensuração da AV final em diferentes diagnósticos pré-operatórios?

As indicações de CP devido a leucoma encontradas no nosso estudo, apesar da pequena amostragem, diferem muito das encontradas nos países desenvolvidos, onde existe um predomínio do ceratocone ${ }^{(1-2)}$ e ceratopatia bolhosa ${ }^{(3)}$, como também no Brasil, que apresentou um maior número de casos de ceratocone ${ }^{(14)}$. Mas encontramos similaridade nas indicações de ceratoplastia penetrantes realizadas na Índia ${ }^{(4)}$, onde observou-se um grande número de casos de leucoma (pós ceratite, pós perfuração e de causa desconhecida), em pacientes com baixo nível sócio-econômico. Neste mesmo estudo, em pacientes com melhor nível sócio-econômico, as maiores indicações de CP foram a ceratopatia bolhosa afácica e pseudofácica. Apesar do nosso estudo não avaliar o status sócioeconômico, existe uma tendência desses pacientes pertencerem a uma classe sócio-econômica baixa, já que nosso hospital oferece serviço gratuito a uma população de baixa renda. Pacientes com baixo nível sócio-econômico têm demonstrado elevado risco de falha no transplante ${ }^{(9)}$, portanto este fator pode ter interferido no resultado da acuidade visual final.

As complicações per e pós-operatórias mais freqüentes em nosso estudo foram a falência do enxerto, extravasamento pela sutura e glaucoma pós-operatório, e estas são similares ao que é descrito na literatura ${ }^{(6)}$. O índice de rejeição do aloenxerto foi similar para todos os grupos com exceção do ceratocone, embora no grupo dos leucomas tenham sido encontradas córneas muito neovascularizadas no pré-operatório, o que representa um fator de alto risco para falência do enxerto ${ }^{(8,10,15)}$.

As nossas indicações de CP diferem das apresentadas nos países desenvolvidos, pelo grande número de casos de leucomas. Talvez exista uma certa tendência nos países desenvolvidos de reservar córneas para pacientes com melhor prognóstico visual, ou seja, pacientes portadores de ceratocone ${ }^{(1)}$.

\section{CONCLUSÃO}

Pelo nosso estudo conclui-se que o ceratocone apresentou um resultado de melhora da AV em linhas significativamente maior que os demais grupos.

\section{AGRADECIMENTOS}

Marcos Moraes - Enfermeiro da Captação de Órgãos do Hospital de Base.

Lewis Joel Greene - Editor Chefe do Brazilian Journal. Pela colaboração na revisão deste artigo.

ABS TRACT

Purpose: This study was designed to evaluate the most frequent indications, as well as the results of final visual acuity (VA) after penetrating keratoplasty (PK), performed by residents. Methods: A consecutive series of 159 cases of PK was followed during the period from January 1991 to October 1998, at the Medical School of São Jose do Rio Preto - "Hospital de Base", in terms of indication for penetrating keratoplasty and final visual acuity. The minimum follow-up to assess the final visual acuity was 6 months. The cases of penetrating keratoplasty were divided into 6 groups of preoperative diagnosis for better comparison and statistical analysis of the final visual acuity. Such groups were: keratoconus, pseudophakic bullous keratopathy, aphakic bullous keratopathy, Fuchs' endothelial distrophy, band keratopathy and leukomas. All surgeries were performed by residents under supervision. Results: Keratoconus was the group that reached the highest average increase of postoperative lines of visual acuity (7.55 \pm $2.83)$ and band keratopathy the smallest one $(0.33 \pm 3.20)$. A significant difference was observed among the indications for penetrating keratoplasty when compared to developed countries, where keratoconus is the most predominant indication. Our analysis revealed that the most important indications for penetrating keratoplasty in decreasing order were: leukoma, pseudophakic bullous keratopathy, keratoconus, Fuchs' endothelial distrophy, aphakic bullous keratopathy and band keratopathy. Conclusion: Keratoconus showed significant difference of final visual acuity levels ( $p$ value $<0,05$ ) when compared to the other 5 groups of penetrating keratoplasty indications.

Keywords: Corneal opacity; Penetrating keratoplasty; Visual acuity; Developing countries; Hospital medical staff

\section{REFERENCIAS}

1. Legeais JM, Labetoulle M, Renard G, Gaillot D, Pouliquen Y. Indications des kératoplasties transfixiantes. Etude retrospective de 2,962 cas sur onze ans. J Fr Ophtalmol 1993;16:516-22.

2. Mamalis N, Anderson CW, Kreisler KR, Lundergan MK, Olson RJ. Changing trends in the indications for penetrating keratoplasty. Arch Ophthalmol 1992;110:1409-11.

3. Damji KF, Rootman J, White V, Dubord PJ, Richards JSF. Changing indications for penetrating keratoplasty in Vancouver, 1978-87. Can J Ophthalmol 1990;25:243-8.

4. Dandona L, Ragu K, Janarthanan M, Naduvilath TJ, Shenoy R, Rao GN. Indications for penetrating keratoplasty in India. Indian J Ophthalmol 1997; 45:163-8. 
5. Bishop VL, Robinson LP, Wechler AW, Billson FA. Corneal graft survival: a retrospective Australian study. Aust N Z J Ophthalmol 1986;14:133-8.

6. Wiggins RE, Cobo M, Foulks GN. Results of penetrating keratoplasty by residents. Arch Ophthalmol 1990;108:851-3.

7. Abbott RL, Forster RK. Determinants of graft clarity in penetrating keratoplasty. Arch Opthalmol 1979;97:1075-9.

8. Dandona L, Naduvilath TJ, Janarthanan M, Ragu K, Rao GN. Survival analysis and visual outcome in a large series of corneal transplants in India. Br J Ophthalmol 1997;81:726-31.

9. Price Jr FW, Whitson WE, Marks RG. Progression of visual acuity after penetrating keratoplasty. Ophthalmology 1991;98:1177-85.

10. Yamagami S, Susuki Y, Tsuru T. Risk factors for graft failure in penetrating keratoplasty. Acta Ophthalmol Scand 1996;74:584-8.
11. Ing JJ, Ing HH, Nelson LR, Hodge DO, Bourne WM. Ten-year postoperative results of penetrating keratoplasty. Ophthalmology 1998;105:1855-65.

12. Bourne WM, Hodge DO, Nelson LR. Corneal endothelium five years after transplantation. Am J Ophthalmol 1994;118:185-96.

13. Kus MM, Seitz B, Langenbucher A, Naumann GOH. Endothelium and pachymetry of clear corneal grafts 15 to 33 years penetrating keratoplasty. Am J Ophthalmol 1999;127:600-2.

14. Dantas MCN, Dantas PEC, Holzchuh N, Lui Netto A, Giovedi Filho R, Giovedi M, Almeida GV. Indicações de transplante penetrante de córnea: 1991-1995. Arq Bras Oftalmol 1998;61:26-33

15. Wilson SE, Kaufman HE. Graft failure after penetrating keratoplasty. [commented on Surv Ophthalmol 1991;35:475-6]. Surv Ophthalmol 1990; $34: 325-56$

\section{ABO ELETRÔNICO}

\section{A versão eletrônica dos Arquivos Brasileiros de} Oftalmologia com textos completos está disponível em:

- http://www.abonet.com.br

- SciELO - Scientific Electronic Library Online -

http://www.scielo.org

- http://www.freemedicaljournals.com 